Research in Astronomy and Astrophysics manuscript no.

(IATEX: RAA-2021-0173.R1.tex; printed on August 29, 2021; 8:26)

\title{
Intra-day variability of BL Lacertae from 2016 to 2018
}

\author{
Tian Li, Jianghua Wu, Nankun Meng, Yan Dai and Xiaoyuan Zhang \\ Department of Astronomy, Beijing Normal University, Beijing 100875, China; jhwu@bnu.edu.cn \\ Received 20xx month day; accepted 20xx month day
}

\begin{abstract}
We monitored BL Lacertae in the B, V, R and I bands for 14 nights during the period of 2016-2018. The source showed significant intraday variability on 12 nights. We performed colour-magnitude analysis and found that the source exhibited bluer-when-brighter chromatism. This bluer-when-brighter behavior is at least partly caused by the larger variation amplitude at shorter wavelength. The variations at different wavelengths are well correlated and show no inter-band time lag.
\end{abstract}

Key words: galaxies: active-BL Lacertae objects: individual: BL Lacertaegalaxies:photometry.

\section{INTRODUCTION}

Blazar is the most violently variable object among all kinds of active galactic nuclei (AGNs). The relativistic jets of blazars are believed to orient close to the line of our sight and powered by the central accretion disk-supermassive black hole systems. BL Lacertae object and flat-spectrum radio quasar are the subsets of blazar. The BL Lacertae object is named after the well-known blazar called BL Lacertae, which is characterized by its high and variable polarization, absence of strong emission lines in the optical spectrum, synchrotron emission from relativistic jets, and intense flux and spectral variability from radio to $\gamma$-ray on a wide variety of time-scales (Wagner \& Witzel 1995; Böttcher et al. 2003). For intraday variability (IDV), the flux can change over hundredth or even tenth magnitudes within several hours (Agarwal \& Gupta 2015). Blazar's spectral energy distribution (SED) displays two peaks (Fossati et al. 1998). We can divide the BL Lacs into three classes based on the locations of these peaks. For the high energy peaked BL Lacs (HBLs), their first peaks locate in UV/X-rays while the second peaks locate at $\mathrm{TeV}$ energies. The synchrotron emission peaks of Intermediate-frequency-peaked BL Lacs (IBL) lies in optical region. BL Lacertae is a low-frequency peaked BL Lacs (LBL) (Ciprini et al. 2004; Abdo et al. 2010) as its first component peaks at infrared while its second component peaks around MeV-GeV (Padovani \& Giommi 1995; Abdo et al. 2010).

BL Lacertae, hosted in a giant elliptical galaxy with $\mathrm{R}=15.5$ (Scarpa et al. 2000), has a redshift of $\mathrm{z}=$ $0.0668 \pm 0.0002$ (Miller \& Hawley 1977). It was once observed in several multiwavelength campaigns car- 
et al. 2009). Some other investigations have been carried out to study its flux variations, spectral changes, and inter-band cross-correlations (Epstein et al. 1972; Carini et al. 1992; Villata et al. 2002; Papadakis et al. 2003; Zhai \& Wei 2012; Agarwal \& Gupta 2015; Gaur et al. 2015; Meng et al. 2017; Bhatta \& Webb 2018; Sadun et al. 2020). Most of the observations found its amplitude of IDV is larger at a shorter wavelength. The IDV amplitude is usually larger when the duration of the observation is longer (Gupta \& Joshi 2005; Gaur et al. 2015, 2017). The IDV amplitude also decreases as the source flux increases (Gaur et al. 2015, 2017). The reason might be that the irregularities in the turbulent jet will decrease when the source is at a bright state and fewer non-axisymmetric bubbles were carried downward in the relativistic jets (Marscher 2014; Gaur et al. 2015). Sandrinelli et al. (2018) found a possible $\gamma$-ray and optical correlated quasi-periodicities of $1.86 \mathrm{yr}$. Bluer-when-brighter (BWB) trend was found in previous observations. The BWB trend tends to appear on short timescales rather than on long timescales, indicating that there are probably two different components in the variability of BL Laccertae (Villata et al. 2002, 2004a). As BL Lac objects usually show BWB trends, redder-when-brighter trends are frequently seen in flat spectrum radio quasar (FSRQs) (Li et al. 2018; Gupta et al. 2019). The variation in different bands are highly correlated. Several authors found time-lag between variations in different bands of BL Lacertae. For example, Papadakis et al. (2003) found a delay of $0.4 \mathrm{~h}$ between the B and I bands. Hu et al. (2006) found a delay of 11.6 minutes between the e and $\mathrm{m}$ bands. A possible time lag of 11.8 minutes between the $\mathrm{R}$ and $\mathrm{V}$ bands was reported by Meng et al. (2017).

In this paper, we aim to study the optical IDV and spectral variations of BL Lac. We carried out photometric measurement of this object on 14 nights in 2016-2018. We also tried to find any possible inter-band time lags in order to study the physical nature of the acceleration and cooling mechanisms in the relativistic jet and the origin of IDV. The paper is organized as follows. In section 2, we describe our observations and data reductions. Section 3 describes the analysis techniques followed by results. Section 4 gives the conclusions.

\section{OBSERVATIONS AND DATA REDUCTIONS}

The observations were carried out on 14 nights in the period from 2016 November 3 to 2018 December 3. We used an $85 \mathrm{~cm}$ reflector to do the observations. It is at Xinglong Station, National Astronomical Observatories, Chinese Academy of Science (NAOC). The telescope uses primary focus system (F/3.27) with an Andor CCD and Johnson and Cousins filters UBVRI. The CCD has $2048 \times 2048$ pixels and the pixel size is $12 \mu \mathrm{m}$.

The photometric observations were performed in the B, V, R and I bands, we chose different combination of filters on different observations (see Table 1). The camera was switched to a cyclical mode for the exposures. In order to get enough signal to noise ratio (SNR), the exposure times were set according to the filter, weather condition, seeing, moon phase, and atmospheric transparency. It ranges from 8 to 120 seconds. The observation log is shown in the Table 1. Figure 1 shows the finding chart.

We used the IRAF to reduce the data. The procedures included bias subtraction, flat fielding, extraction of instrumental aperture magnitude, and flux calibration. The average FWHM of the stellar images varied 
Table 1: Observation log

\begin{tabular}{|c|c|c|c|c|c|}
\hline Julian Date & $\begin{array}{c}\text { Date } \\
\text { ( yyyy mm dd ) }\end{array}$ & Passbands & Data points & $\begin{array}{r}\text { Exposure time } \\
\text { (seconds) }\end{array}$ & $\begin{array}{c}\text { Duration } \\
\text { (hours) }\end{array}$ \\
\hline \multirow[t]{2}{*}{2457696} & 20161103 & $V$ & 160 & 60 & 6.2 \\
\hline & & $R$ & 160 & 60 & 6.2 \\
\hline \multirow[t]{2}{*}{2457697} & 20161104 & $V$ & 94 & 60 & 3.6 \\
\hline & & $R$ & 93 & 60 & 3.6 \\
\hline \multirow[t]{2}{*}{2457699} & 20161106 & $V$ & 178 & 60 & 7.0 \\
\hline & & $R$ & 181 & 60 & 7.0 \\
\hline \multirow[t]{3}{*}{2457745} & 20161222 & $B$ & 97 & 30 & 2.3 \\
\hline & & $V$ & 97 & 20 & 2.3 \\
\hline & & $R$ & 95 & 8 & 2.2 \\
\hline \multirow[t]{3}{*}{2457746} & 20161223 & $B$ & 103 & 40 & 2.8 \\
\hline & & $V$ & 101 & 18 & 2.8 \\
\hline & & $R$ & 101 & 10 & 2.8 \\
\hline \multirow[t]{3}{*}{2458012} & 20170915 & $B$ & 232 & 60 & 8.3 \\
\hline & & $R$ & 250 & 20 & 8.5 \\
\hline & & $I$ & 233 & 20 & 8.3 \\
\hline \multirow[t]{3}{*}{2458013} & 20170916 & $B$ & 235 & 60 & 8.2 \\
\hline & & $R$ & 235 & 20 & 8.4 \\
\hline & & $I$ & 232 & 20 & 8.1 \\
\hline \multirow[t]{3}{*}{2458014} & 20170917 & $B$ & 230 & 60 & 8.3 \\
\hline & & $R$ & 236 & 20 & 8.5 \\
\hline & & $I$ & 228 & 20 & 8.3 \\
\hline \multirow[t]{4}{*}{2458369} & 20180907 & $B$ & 31 & 60 & 2.3 \\
\hline & & $V$ & 31 & 60 & 2.3 \\
\hline & & $R$ & 31 & 60 & 2.3 \\
\hline & & $I$ & 31 & 60 & 2.3 \\
\hline \multirow[t]{4}{*}{2458370} & 20180908 & $B$ & 52 & 60 & 3.8 \\
\hline & & $V$ & 52 & 60 & 3.8 \\
\hline & & $R$ & 52 & 60 & 3.8 \\
\hline & & $I$ & 52 & 60 & 3.8 \\
\hline \multirow[t]{3}{*}{2458420} & 20181028 & $B$ & 67 & 60 & 5.1 \\
\hline & & $V$ & 68 & 60 & 5.1 \\
\hline & & $R$ & 68 & 60 & 5.0 \\
\hline \multirow[t]{2}{*}{2458424} & 20181101 & $R$ & 132 & 50 & 4.2 \\
\hline & & $I$ & 132 & 40 & 4.2 \\
\hline \multirow[t]{3}{*}{2458425} & 20181102 & $B$ & 84 & 20 & 3.5 \\
\hline & & $V$ & 84 & 40 & 3.5 \\
\hline & & $R$ & 84 & 60 & 3.5 \\
\hline \multirow[t]{3}{*}{2458456} & 20181203 & $B$ & 51 & 120 & 4.3 \\
\hline & & $V$ & 51 & 60 & 4.3 \\
\hline & & $R$ & 51 & 60 & 4.2 \\
\hline
\end{tabular}

aperture size of 1.5 times of the average Full width at half maximum (FWHM) of the stellar images. The inner and outer radii of the sky annuli were adopted as 5 and 7 times of the stellar FWHM, respectively. The magnitudes of BL Lacertae were calibrated with respect to the magnitude of star 3 in Figure 1. star 6 is 


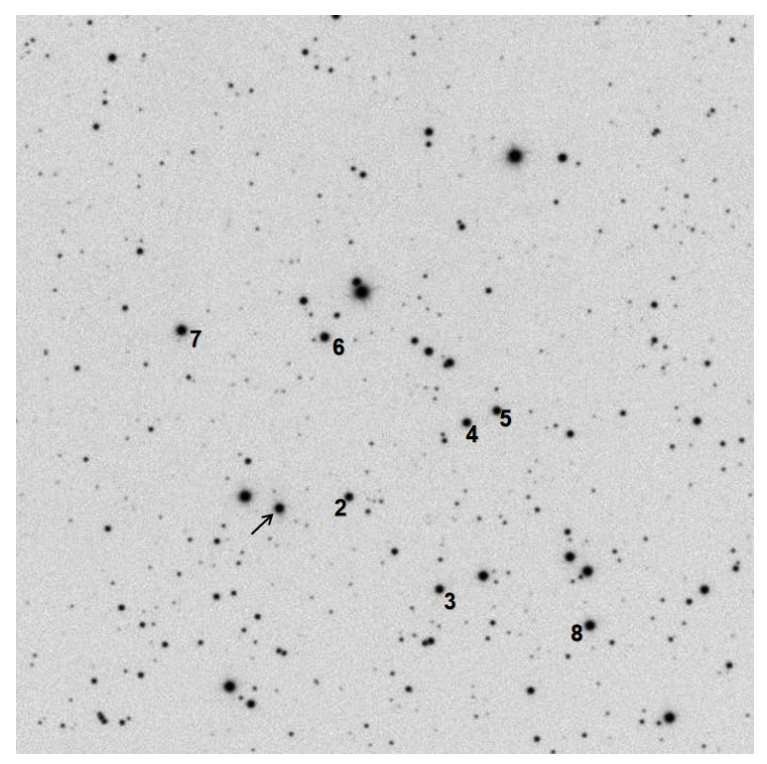

Fig. 1: Finding chart of BL Lacertae in $R$ band. The images was taken on 2016 November 03. The BL Lacertae is marked by an arrow. Star 6 is the check star, Star 2 and Star 4-8 were selected and used in the quantitative assessment of the IDV. Their magnitudes were all calibrated relative to the brightness of star 3 .

observations. Star 2 and stars 4-8 were selected and used in the quantitative assessment of the IDV, as will be described in the next section. Their magnitudes were calibrated relative to the brightness of star 3 . The standard magnitudes of stars 3, 4, and 6 in the B, V, R and I bands are given by Smith et al. (1985).

The photometric errors from IRAF are significantly underestimated according to Goyal et al. (2013). Their method is to determine a coefficient $\eta$ which is the ratio between the real photometric error and that given by IRAF. Here we selected the check star (star 6) due to its lowest fluctuations and calculate $\chi$ by using the equation

$$
\chi^{2}=\sum_{i=1}^{N} \frac{\left(V_{i}-\bar{V}\right)^{2}}{\sigma_{i}^{2}}
$$

In this equation, $V_{i}$ is the ith differential magnitude, $\bar{V}$ is the mean of all differential magnitudes and $\sigma_{i}$ is the original error given by IRAF. The degree of freedom $\nu$ can be calculated from:

$$
\nu=N-1=\chi^{2} / \eta^{2}
$$

Then we obtained the regression analysis with fixed slope to calculate the coefficient $\eta$. See Goyal et al. (2013) for more details.

We calculated the $\eta$ of each band in each day and find that the $\eta$ ranges from 1.0764 to 1.7367 , which is used to modify the original errors obtained by IRAF.

\section{RESULTS}

\subsection{Light curves}

The overall light curves are displayed in Figure 2. The light curves show obvious long term fluctuations. 


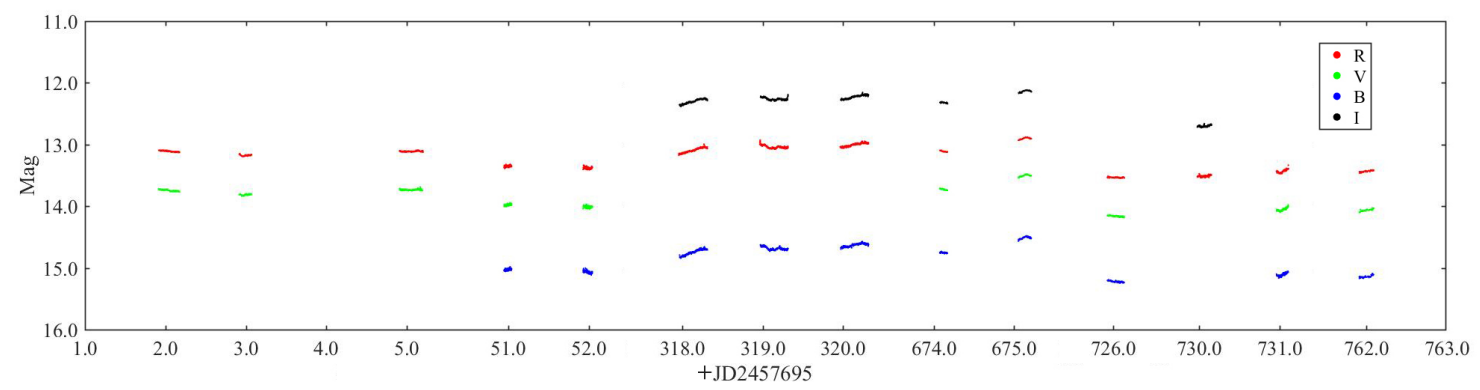

Fig. 2: Light curves of BL Lacertae in the B, V, R and I bands in 3 years. Different colour dots represent data in different bands.

The intra-night light curves of the object are plotted in Figure 3. Heidt \& Wagner (1996) developed a method to quantify the IDV amplitude:

$$
A=\sqrt{\left(m_{\max }-m_{\min }\right)^{2}-2 \sigma^{2}},
$$

where $\sigma$ is the measurement error. According to equation (3), the value $A$ will always be larger than 0 , however it does not means that the corresponding light curve is variable. The most violent variation happened on JD2458012 (2017 September 15) when the IDV amplitude reached 16.5\% (0.17 mag) in the B band and the variation rate was $0.3 \mathrm{mag} / \mathrm{hr}$. On JD2458014 (2017 September 17) the object reached its brightest state of $\mathrm{R}=12.95$, while on JD2458420 (2018 October 28) the object was at its faintest state with $\mathrm{R}=13.55$. The IDV amplitude for each band in each night are included in Table 2. Figure 4 shows the IDV amplitudes of the variable light curves. According to Figure 4 and Table 2, the IDV amplitude is greater in higher energy bands. The IDV amplitude is comparable in a few cases where the differences between IDV amplitude are smaller than $0.5 \%$. This trend has also been observed by others (e.g. Nesci et al. 1998; Webb et al. 1998; Fan \& Lin 1999; Nikolashvili \& Kurtanidze 2004; Meng et al. 2017).

\subsection{Variability Detection}

We performed the nested analysis of variance (ANOVA) and enhanced F-test to examine and to quantify the intraday variability (de Diego et al. 2015).

In the nested ANOVA analysis, we separated the data points of a certain band on a certain day into groups, with 5 data points in each group. The null hypothesis is that the deviation of the mean values of differential light curves in each group is zero. The expressions of the degrees of freedom and F-value are shown in the Equation 4 in de Diego et al. (2015).

The enhanced F-test uses several comparison field stars. First, the errors of the field stars are scaled into our target's level. By fitting an exponential curves to our comparison stars we can obtain the relationship between the standard deviation and the mean magnitude of the light curve. This relationship can be used to transfer the error of field stars into the level of BL Lacertae. At last we subtract the mean magnitude from each field star's light curve and stack them together. The F value is the variance of BL Lacertae's curve 

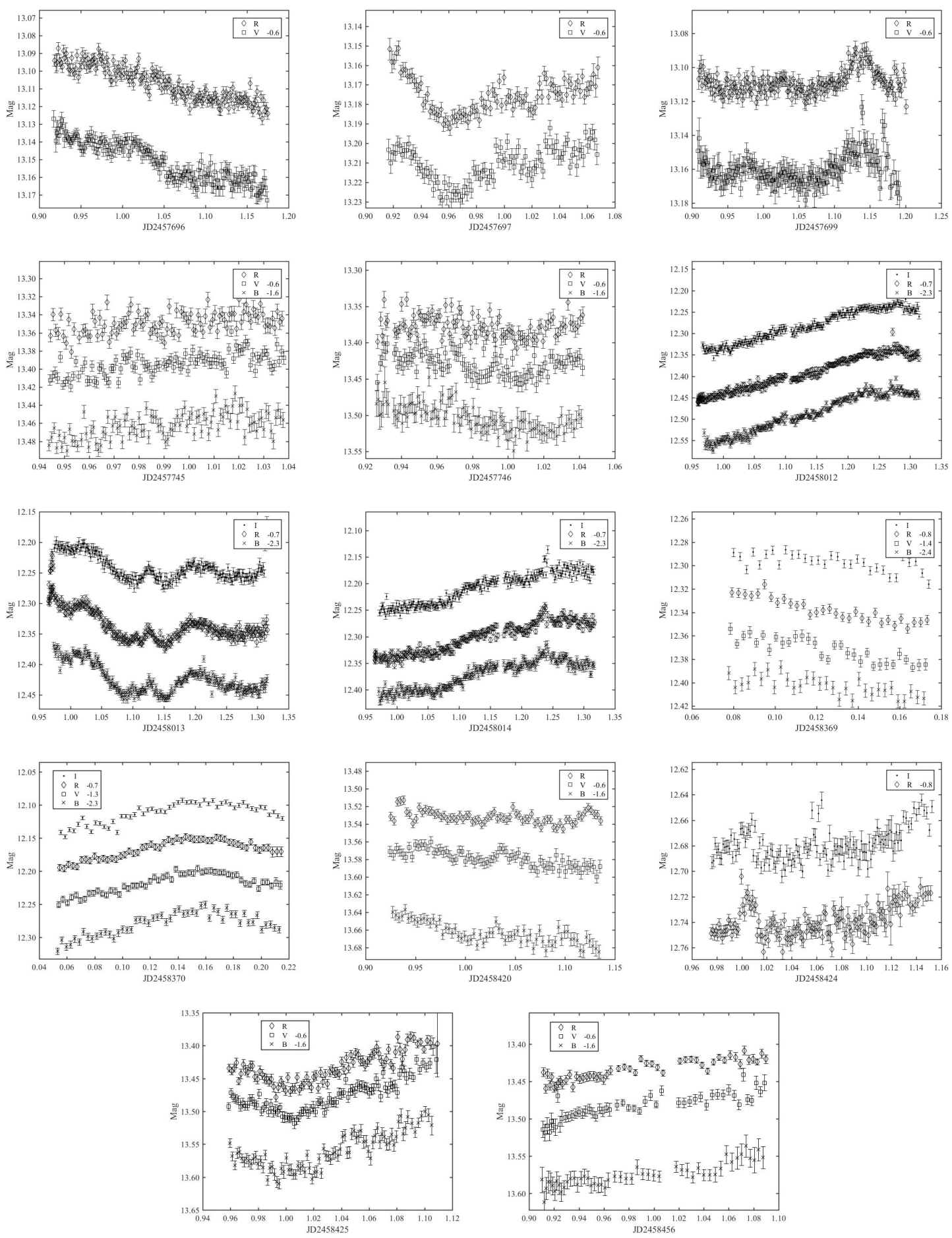

Fig. 3: Intraday light curves. For clarity, the B, V, R, and I light curves are shifted. The shifted magnitudes are given in the plots.

\subsection{Variations Result}

The results of the two tests are listed in Table 2. $\nu 1$ and $\nu 2$ are degrees of freedom in each test. Column (15) is the IDV amplitude. Column (14) is the Variability. $F_{\text {crit }}$ is the critical value of F-test at $\alpha=0.05$ ( $\alpha$ is significance level). When the light curve's F-value is larger than $\mathrm{F}_{\text {crit }}$ and its P-value is smaller than 


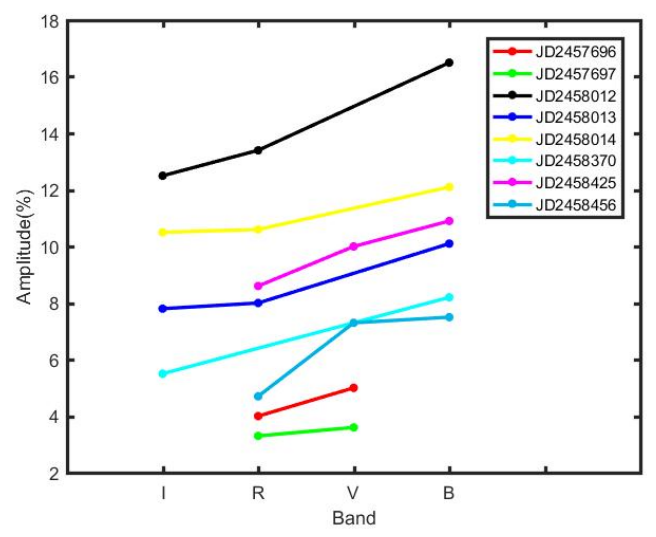

Fig. 4: The IDV amplitudes of the variable light curves

'Y'(Yes). 'N'(No) means the light curve did not pass at least one of the tests. Twenty-three light curves on twelve nights are variable according to both tests, while three didn't pass both tests. Fourteen light curves passed one of the tests while their P-value of the other test is larger than 0.05 , we can not determine their variability, so we mark them as 'P' (Possible). Small flares can be seen in the $V$ band on 2016 November 4 (JD2457697) and 2016 November 06 (JD2457699), and all bands on 2018 October 28 (JD2458420). Due to the measurement error, these light curves only passed one of the tests but are still very likely variable.

\subsection{Inter-band correlation analysis and time lags}

To search for the possible time lags between variations in different bands, we performed cross-correlation analyses. Two cross-correlation methods are used. One is the z-transformed discrete correlation functions (ZDCFs) (Alexander 1997, 2013), in which equal population binning and Fisher's z-transform are used to correct several biases of the discrete correlation function of Edelson \& Krolik (1988). A Gaussian Fitting (GF) of the ZDCF points greater than $75 \%$ of the peak value can give an estimate of the time lag and the associated errors. However, Gaussian fitting may underestimate the error (e.g. Wu et al. 2012). So we only take the results of $\mathrm{ZDCF}+\mathrm{GF}$ method as a reference. For more reliable estimate of the time lags and errors, we used the interpolated cross-correlation function (ICCF)(Gaskell \& Peterson 1987). Peterson et al. (1998, 2004) employed a Monte-Carlo (MC) method to calculate the centroid position of the ICCF and its error. Flux-randomization (FR) and random-subset selection (RSS) are applied in each Monte-Carlo realization. Here we performed 5000 Monte-Carlo realizations. The results are listed in Table 3. On 2018 December 3 (JD2458456), the Gaussian fitting failed to fit the ZDCF, the results are not listed in the table.

According to our results, no time lag has significance greater than $3 \sigma$, thus we failed to detect any time lags at high significance in our observations.

\subsection{Color Variations}

By calculating the colour indices of $B-R, V-R, V-I$, and $B-I$, we investigated the color variation with respect to the magnitude of BL Lacertae for each night. A linear fit was made to the data points. 
Table 2: IDV test results.

\begin{tabular}{|c|c|c|c|c|c|c|c|c|c|c|c|c|c|c|}
\hline \multirow{3}{*}{$\begin{array}{c}\text { Julian Date } \\
\text { (1) }\end{array}$} & \multirow{3}{*}{$\begin{array}{c}\text { Date } \\
\text { ( yyyy mm dd ) } \\
\text { (2) }\end{array}$} & \multirow{3}{*}{$\begin{array}{l}\text { Filter } \\
\text { (3) }\end{array}$} & \multicolumn{5}{|c|}{ Enhanced F-test } & \multicolumn{5}{|c|}{ Nested ANOVA } & \multirow{3}{*}{$\begin{array}{c}\text { Variability } \\
\text { (14) }\end{array}$} & \multirow{3}{*}{$\begin{array}{r}\text { Amplitude } \\
(\%) \\
(15)\end{array}$} \\
\hline & & & \multirow{2}{*}{$\begin{array}{l}F \\
(4)\end{array}$} & \multirow{2}{*}{$\begin{array}{l}\nu_{1} \\
(5)\end{array}$} & \multirow{2}{*}{$\begin{array}{l}\nu_{2} \\
\text { (6) }\end{array}$} & \multirow{2}{*}{$\begin{array}{r}F_{\text {crit }} \\
\text { (7) }\end{array}$} & $\begin{array}{l}\mathrm{P} \\
\end{array}$ & \multirow{2}{*}{$\begin{array}{l}F \\
\text { (9) }\end{array}$} & \multirow{2}{*}{$\begin{array}{c}\nu_{1} \\
(10)\end{array}$} & \multirow{2}{*}{$\begin{array}{c}\nu_{2} \\
(11)\end{array}$} & \multirow{2}{*}{$\begin{array}{r}F_{\text {crit }} \\
(12)\end{array}$} & \multirow{2}{*}{$\begin{array}{l}\mathrm{P} \\
\quad(13)\end{array}$} & & \\
\hline & & & & & & & (8) & & & & & & & \\
\hline \multirow[t]{2}{*}{2457696} & 20161103 & $V$ & 3.67 & 160 & 800 & 1.21 & $<0.0001$ & 5.10 & 31 & 159 & 1.83 & $<0.0001$ & $\mathrm{Y}$ & 5.0 \\
\hline & & $R$ & 5.89 & 160 & 800 & 1.21 & $<0.0001$ & 2.46 & 31 & 159 & 1.83 & $<0.0001$ & $\mathrm{Y}$ & 4.0 \\
\hline \multirow[t]{2}{*}{2457697} & 20161104 & $V$ & 3.02 & 94 & 470 & 1.28 & $<0.0001$ & 1.92 & 17 & 89 & 2.21 & $<0.0001$ & $\mathrm{Y}$ & 3.6 \\
\hline & & $R$ & 6.52 & 93 & 465 & 1.29 & $<0.0001$ & 3.79 & 17 & 89 & 2.21 & $<0.0001$ & $\mathrm{Y}$ & 3.3 \\
\hline \multirow[t]{2}{*}{2457699} & 20161106 & $V$ & 1.08 & 178 & 890 & 1.21 & $<0.0001$ & 2.28 & 18 & 94 & 1.79 & 0.0082 & $\mathrm{~N}$ & \\
\hline & & $R$ & 0.11 & 181 & 905 & 1.25 & 0.9999 & 1.51 & 19 & 99 & 1.77 & 0.1044 & $\mathrm{~N}$ & \\
\hline 2457745 & 20161222 & $B$ & 1.89 & 97 & 485 & 1.28 & $<0.0001$ & 1.14 & 18 & 94 & 2.21 & 0.3297 & $P$ & 5.4 \\
\hline & & $V$ & 2.80 & 97 & 485 & 1.28 & $<0.0001$ & 0.48 & 18 & 94 & 2.21 & 0.9584 & $P$ & 4.1 \\
\hline & & $R$ & 2.06 & 95 & 475 & 1.28 & $<0.0001$ & 1.06 & 18 & 94 & 2.21 & 0.4118 & $\mathrm{P}$ & 7.5 \\
\hline 2457746 & 20161223 & $B$ & 1.36 & 103 & 515 & 1.27 & 0.0165 & 3.08 & 19 & 99 & 2.12 & $<0.0001$ & $\mathrm{Y}$ & 10.6 \\
\hline & & $V$ & 2.10 & 101 & 505 & 1.27 & $<0.0001$ & 1.29 & 19 & 99 & 2.12 & 0.0686 & $\mathrm{P}$ & 7.5 \\
\hline & & $R$ & 2.28 & 101 & 505 & 1.27 & $<0.0001$ & 1.41 & 19 & 99 & 2.12 & 0.5124 & $\mathrm{P}$ & 7.4 \\
\hline 2458012 & 20170915 & $B$ & 20.15 & 232 & 1160 & 1.18 & $<0.0001$ & 8.46 & 45 & 229 & 1.66 & $<0.0001$ & $\mathrm{Y}$ & 16.5 \\
\hline & & $R$ & 28.59 & 250 & 1250 & 1.17 & $<0.0001$ & 8.07 & 49 & 249 & 1.63 & $<0.0001$ & Y & 13.4 \\
\hline & & $I$ & 28.69 & 233 & 1165 & 1.18 & $<0.0001$ & 7.43 & 45 & 229 & 1.66 & $<0.0001$ & $\mathrm{Y}$ & 12.5 \\
\hline 2458013 & 20170916 & $B$ & 7.99 & 235 & 1175 & 1.17 & $<0.0001$ & 3.33 & 46 & 234 & 1.66 & $<0.0001$ & $\mathrm{Y}$ & 10.1 \\
\hline & & $R$ & 13.87 & 255 & 1275 & 1.17 & $<0.0001$ & 3.61 & 50 & 254 & 1.63 & $<0.0001$ & $\mathrm{Y}$ & 8.0 \\
\hline & & $I$ & 6.14 & 232 & 1160 & 1.18 & $<0.0001$ & 3.62 & 45 & 229 & 1.66 & $<0.0001$ & $\mathrm{Y}$ & 7.8 \\
\hline 2458014 & 20170917 & $B$ & 6.46 & 230 & 1150 & 1.18 & $<0.0001$ & 2.45 & 45 & 229 & 1.66 & $<0.0001$ & $\mathrm{Y}$ & 12.1 \\
\hline & & $R$ & 12.56 & 236 & 1180 & 1.17 & $<0.0001$ & 2.74 & 46 & 234 & 1.66 & $<0.0001$ & $\mathrm{Y}$ & 10.6 \\
\hline & & $I$ & 16.54 & 228 & 1140 & 1.18 & $<0.0001$ & 4.89 & 44 & 224 & 1.68 & $<0.0001$ & $\mathrm{Y}$ & 10.5 \\
\hline 2458369 & 20180907 & $B$ & 1.02 & 31 & 155 & 1.53 & 0.4436 & 1.56 & 5 & 29 & 2.62 & 0.2106 & $\mathrm{~N}$ & \\
\hline & & $V$ & 4.44 & 31 & 155 & 1.53 & $<0.0001$ & 2.02 & 5 & 29 & 2.62 & 0.1123 & $P$ & 3.3 \\
\hline & & $R$ & 4.76 & 31 & 155 & 1.53 & $<0.0001$ & 6.94 & 5 & 29 & 2.62 & 0.0003 & $\mathrm{Y}$ & 3.2 \\
\hline & & $I$ & 4.88 & 31 & 155 & 1.53 & $<0.0001$ & 1.25 & 5 & 29 & 2.62 & 0.3176 & $\mathrm{P}$ & 3.3 \\
\hline 2458370 & 20180908 & $B$ & 8.00 & 52 & 260 & 1.40 & $<0.0001$ & 4.29 & 9 & 49 & 2.12 & 0.0006 & $\mathrm{Y}$ & 8.2 \\
\hline & & V & 8.66 & 52 & 260 & 1.40 & $<0.0001$ & 1.96 & 9 & 49 & 2.12 & 0.0706 & $P$ & 5.6 \\
\hline & & $R$ & 14.22 & 52 & 260 & 1.40 & $<0.0001$ & 1.59 & 9 & 49 & 2.12 & 0.1523 & $P$ & 4.9 \\
\hline & & $I$ & 27.30 & 52 & 260 & 1.40 & $<0.0001$ & 3.50 & 9 & 49 & 2.12 & 0.0028 & $\mathrm{Y}$ & 5.5 \\
\hline 2458420 & 20181028 & $B$ & 2.37 & 67 & 335 & 1.34 & $<0.0001$ & 1.66 & 12 & 64 & 1.94 & 0.1040 & $P$ & 5.6 \\
\hline & & $V$ & 3.01 & 68 & 340 & 1.34 & $<0.0001$ & 1.49 & 12 & 64 & 1.94 & 0.1597 & $\mathrm{P}$ & 4.4 \\
\hline & & $R$ & 3.81 & 68 & 340 & 1.34 & $<0.0001$ & 0.99 & 12 & 64 & 1.94 & 0.4700 & $P$ & 3.3 \\
\hline 2458424 & 20181101 & $R$ & 2.64 & 132 & 660 & 1.24 & $<0.0001$ & 1.09 & 25 & 129 & 1.94 & 0.3716 & $\mathrm{P}$ & 6.3 \\
\hline & & $I$ & 3.87 & 132 & 660 & 1.24 & $<0.0001$ & 3.93 & 25 & 129 & 1.94 & 0.0008 & $\mathrm{Y}$ & 6.4 \\
\hline 2458425 & 20181102 & $B$ & 4.31 & 84 & 420 & 1.30 & $<0.0001$ & 2.82 & 15 & 79 & 2.31 & 0.0020 & $\mathrm{Y}$ & 10.9 \\
\hline & & $V$ & 7.12 & 84 & 420 & 1.30 & $<0.0001$ & 4.28 & 15 & 79 & 2.31 & $<0.0001$ & $\mathrm{Y}$ & 10.0 \\
\hline & & $R$ & 7.28 & 84 & 420 & 1.30 & $<0.0001$ & 2.50 & 15 & 79 & 2.31 & 0.0046 & $\mathrm{Y}$ & 8.6 \\
\hline 2458456 & 20181203 & $B$ & 1.52 & 51 & 255 & 1.40 & 0.0195 & 6.27 & 9 & 49 & 2.12 & $<0.0001$ & $\mathrm{Y}$ & 7.5 \\
\hline & & $V$ & 7.32 & 51 & 255 & 1.40 & $<0.0001$ & 4.99 & 9 & 49 & 2.12 & 0.002 & $\mathrm{Y}$ & 7.3 \\
\hline & & $R$ & 7.73 & 51 & 255 & 1.40 & $<0.0001$ & 2.25 & 9 & 49 & 2.12 & 0.0382 & $\mathrm{Y}$ & 4.7 \\
\hline
\end{tabular}
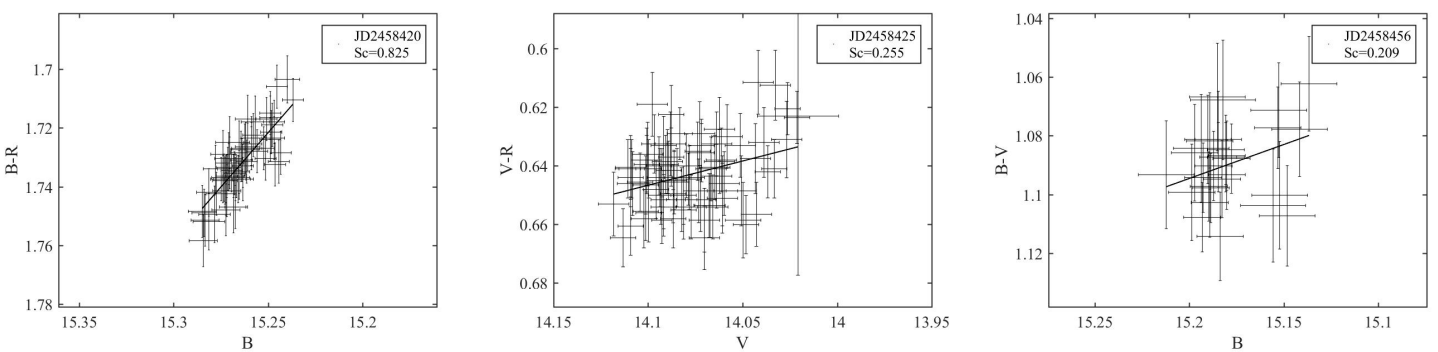

Fig. 5: Examples of intranight colour-magnitude diagrams, which illustrate strong, mild and weak correlations, respectively. 
Table 3: Time lags from correlation analyses

\begin{tabular}{|c|c|c|c|c|}
\hline Julian Date & $\begin{array}{c}\text { Date } \\
\text { ( yyyy mm dd ) }\end{array}$ & Passbands & $\begin{array}{c}\text { ZDCF-GF } \\
\text { (minutes) }\end{array}$ & $\begin{array}{c}\text { ICCF-FR/RSS } \\
\text { (minutes) }\end{array}$ \\
\hline 2457696 & 20161103 & $R-V$ & $-28.86 \pm 9.13$ & $-22.62 \pm 29.01$ \\
\hline 2457697 & 20161103 & $R-V$ & $-7.07 \pm 5.29$ & $-5.73 \pm 6.18$ \\
\hline 2457699 & 20161106 & $R-V$ & $-15.59 \pm 4.49$ & $-5.69 \pm 5.05$ \\
\hline \multirow[t]{3}{*}{2457745} & 20161222 & $R-V$ & $-8.02 \pm 10.63$ & $-6.03 \pm 38.42$ \\
\hline & & $R-B$ & $-11.68 \pm 7.67$ & $-18.56 \pm 36.13$ \\
\hline & & $V-B$ & $-3.90 \pm 1.94$ & $-4.08 \pm 25.57$ \\
\hline \multirow[t]{3}{*}{2457746} & 20161223 & $R-V$ & $-2.77 \pm 4.78$ & $-2.14 \pm 9.12$ \\
\hline & & $R-B$ & $+7.49 \pm 4.34$ & $+10.67 \pm 13.58$ \\
\hline & & $V-B$ & $+10.40 \pm 6.24$ & $+12.45 \pm 12.76$ \\
\hline \multirow[t]{3}{*}{2458012} & 20170915 & $R-I$ & $-7.63 \pm 3.35$ & $-3.34 \pm 4.54$ \\
\hline & & $R-B$ & $-23.87 \pm 5.24$ & $-2.61 \pm 6.18$ \\
\hline & & $I-B$ & $-0.38 \pm 3.16$ & $+2.22 \pm 3.51$ \\
\hline \multirow[t]{3}{*}{2458013} & 20170916 & $R-I$ & $+4.05 \pm 1.29$ & $-1.63 \pm 4.45$ \\
\hline & & $R-B$ & $+2.54 \pm 1.05$ & $+0.13 \pm 3.93$ \\
\hline & & $I-B$ & $+2.00 \pm 0.74$ & $+2.85 \pm 2.63$ \\
\hline \multirow[t]{3}{*}{2458014} & 20170917 & $R-I$ & $-2.25 \pm 2.92$ & $+5.59 \pm 6.88$ \\
\hline & & $R-B$ & $-9.74 \pm 2.63$ & $-3.86 \pm 8.64$ \\
\hline & & $I-B$ & $-0.44 \pm 8.97$ & $-7.13 \pm 5.92$ \\
\hline \multirow[t]{3}{*}{2458369} & 20180907 & $B-R$ & $-15.37 \pm 7.08$ & $-15.11 \pm 29.98$ \\
\hline & & $B-V$ & $-4.68 \pm 10.73$ & $-3.62 \pm 32.65$ \\
\hline & & $B-I$ & $+11.53 \pm 5.33$ & $+7.02 \pm 36.80$ \\
\hline \multirow[t]{3}{*}{2458370} & 20180908 & $B-R$ & $+2.20 \pm 4.78$ & $+1.30 \pm 7.27$ \\
\hline & & $B-V$ & $+1.56 \pm 4.36$ & $+0.41 \pm 7.47$ \\
\hline & & $B-I$ & $+6.15 \pm 5.5$ & $+3.04 \pm 6.52$ \\
\hline \multirow[t]{3}{*}{2458420} & 20181028 & $R-V$ & $+33.49 \pm 9.74$ & $+20.02 \pm 91.25$ \\
\hline & & $R-B$ & $+19.22 \pm 6.94$ & $+16.63 \pm 92.97$ \\
\hline & & $V-B$ & $-16.86 \pm 11.63$ & $-57.09 \pm 66.31$ \\
\hline 2458424 & 20181101 & $R-I$ & $-5.4 \pm 4.05$ & $-0.58 \pm 3.21$ \\
\hline \multirow[t]{3}{*}{2458425} & 20181102 & $R-V$ & $-2.39 \pm 1.07$ & $-1.70 \pm 6.34$ \\
\hline & & $R-B$ & $-4.47 \pm 1.16$ & $+1.69 \pm 6.36$ \\
\hline & & $V-B$ & $-2.87 \pm 1.41$ & $-1.20 \pm 6.56$ \\
\hline \multirow[t]{3}{*}{2458456} & 20181203 & $R-V$ & & $+28.94 \pm 53.46$ \\
\hline & & $R-B$ & & $-10.22 \pm 33.68$ \\
\hline & & $V-B$ & & $+19.81 \pm 46.77$ \\
\hline
\end{tabular}

vertical-axis are fixed as 0.2 and 0.1 mags, respectively in order to make comparison conveniently between panels.

We calculated the Spearman correlation coefficient $(\mathrm{Sc})$ and the corresponding $\mathrm{P}$ value. The results are displayed in Table 4. The first and second column are the date of observation, the third and fourth column are the passbands of color index, the fifth column is the number of datapoints followed by Sc value and corresponding $\mathrm{P}$ value. The last column the the strength of correlation. The bands without variation are not shown in this table because it is pointless to discuss their trend. The numbers of data points of our 
significance level of 0.01 and degree of freedom of 31. Most of our Sc value of color-magnitude diagrams are above 0.344 with $\mathrm{P}$ value lower than 0.0001, indicating strong correlations. On 2017 September 17 (JD2458014), the Sc values of all the color-magnitude diagram are lower than the critical values with $\mathrm{P}$ values larger than 0.01, we take them as no correlations. On 2018 October 28 (JD2458420) the Sc value of $\mathrm{B}-\mathrm{R}$ diagram is 0.820 with the $\mathrm{P}$ value significantly smaller than 0.01 , indicating a strong BWB color behavior. For the rest, the Sc value ranges from 0.4 to 0.6 with $\mathrm{P}$ value smaller than 0.001 . In the B and R bands of 2018 December 3 (JD2458456), R and V bands of 2018 December 3 (JD2458456), the light curves are not correlated.

According to Ikejiri et al. (2011), we do not know if the BWB trend is universal in blazars. There are two types of blazars, BL Lacertae objects and FSRQs. The BWB trend is often observed in BL Lac objects while the RWB behavior is frequently detected in FSRQs (e.g. Gu et al. 2006; Zhang et al. 2018; Li et al. 2018; Gupta et al. 2019). Villata et al. (2002, 2004a) argued that the BWB relation was more likely to be detected in short isolated outbursts. The BWB trend of BL Lacertae has been detected in a number of previous research (Racine 1970; Speziali \& Natali 1998; Vagnetti \& Trevese 2003; Stalin et al. 2006; Papadakis et al. 2007; Ikejiri et al. 2011; Gaur et al. 2015; Wierzcholska et al. 2015; Meng et al. 2018). Our intraday color-magnitude results consist with most of the historical observations.

\section{DISCUSSIONS AND CONCLUSIONS}

We monitored BL Lacertae in the $B, V, R$ and $I$ bands for 14 nights during 2016-2018. The object showed IDV in 23 light curves on 12 nights.

It has been found that the IDV amplitude of BL Lacertae is greater at higher frequencies (e.g. Kurtanidze et al. 2001; Fan et al. 2001; Papadakis et al. 2003; Hu et al. 2006; Gaur et al. 2015; Meng et al. 2017). This behavior has also been detected in our observation. Butuzova (2021) found similar behavior for S5 0716+714. Gaur et al. (2015) interpreted this behavior as higher energy electrons accelerated by shock front lose energy faster than low energy electrons through synchrotron radiation. Hence the amount of higher frequency photons produced by these electrons will have a more violent change than the lower frequency photons. This will be observed as IDV amplitude is greater at higher frequencies. The higher energy electron is produced in a thin layer behind the shock front and lower-frequency emission is spread out behind the shock front (Marscher \& Gear 1985), it results in time lags of the peak of the light curve toward lower frequencies. However, this trend is not universal in every observation. The flux of bluer bands is lower than redder bands and has higher errors than redder bands (Figure 3 shows the magnitude of $B$ band is larger than R band for BL Lacertae). The error component in equation 3 will reduce the IDV amplitude, so the IDV amplitude of bluer bands with higher errors will be reduced more than the IDV amplitude of redder bands. For example on 2017 September 16th (JD2458013), R and I have comparable IDV amplitudes.

BWB trend was found in our observation. This is consistent with previous (e.g. Vagnetti \& Trevese 2003; Gaur et al. 2015; Wierzcholska et al. 2015; Meng et al. 2017; Zhang et al. 2018; Gaur et al. 2017; Bhatta \& Webb 2018; Zhai \& Wei 2012). We did not subtract the contribution of host galaxy from the total flux since Villata et al. (2002) concluded that the color changes are intrinsic property of fast flares and are 
Table 4: The result of Colour-magnitude diagrams.

\begin{tabular}{|c|c|c|c|c|c|c|c|}
\hline Julian Date & $\begin{array}{c}\text { Date } \\
\text { ( yyyy mm dd ) }\end{array}$ & Color index & Magnitude & No. & $\mathrm{Sc}$ & $\mathrm{P}$ & correlation \\
\hline 2457696 & 20161103 & $R-V$ & $V$ & 160 & 0.50 & $6.6 \times 10^{-12}$ & strong \\
\hline 2457697 & 20161104 & $R-V$ & $V$ & 93 & 0.43 & $1.3 \times 10^{-5}$ & strong \\
\hline \multirow[t]{3}{*}{2457745} & 20161222 & $R-V$ & $V$ & 95 & 0.48 & $6.3 \times 10^{-7}$ & strong \\
\hline & & $R-B$ & $B$ & 95 & 0.64 & $1.3 \times 10^{-12}$ & strong \\
\hline & & $V-B$ & $B$ & 97 & 0.64 & $1.5 \times 10^{-12}$ & strong \\
\hline \multirow[t]{3}{*}{2457746} & 20161223 & $R-V$ & $V$ & 101 & 0.54 & $6.5 \times 10^{-9}$ & strong \\
\hline & & $R-B$ & $B$ & 101 & 0.66 & $4.6 \times 10^{-14}$ & strong \\
\hline & & $V-B$ & $B$ & 101 & 0.54 & $3.0 \times 10^{-9}$ & strong \\
\hline \multirow[t]{3}{*}{2458012} & 20170915 & $I-R$ & $R$ & 233 & 0.26 & $6.9 \times 10^{-5}$ & strong \\
\hline & & $R-B$ & $B$ & 232 & 0.62 & $4.5 \times 10^{-26}$ & strong \\
\hline & & $I-B$ & $B$ & 232 & 0.71 & $1.0 \times 10^{-36}$ & strong \\
\hline \multirow[t]{3}{*}{2458013} & 20170916 & $I-R$ & $R$ & 232 & 0.36 & $1.2 \times 10^{-8}$ & strong \\
\hline & & $R-B$ & $B$ & 235 & 0.46 & $7.1 \times 10^{-14}$ & strong \\
\hline & & $I-B$ & $B$ & 235 & 0.57 & $4.1 \times 10^{-21}$ & strong \\
\hline \multirow[t]{3}{*}{2458014} & 20170917 & $I-R$ & $I$ & 228 & 0.12 & $7.9 \times 10^{-2}$ & no \\
\hline & & $R-B$ & $B$ & 230 & 0.04 & $5.6 \times 10^{-1}$ & no \\
\hline & & $I-B$ & $B$ & 228 & 0.04 & $5.7 \times 10^{-1}$ & no \\
\hline 2458369 & 20180907 & $B-I$ & $B$ & 31 & 0.65 & $1.0 \times 10^{-4}$ & strong \\
\hline \multirow[t]{2}{*}{2458370} & 20180908 & $B-R$ & $B$ & 52 & 0.62 & $9.5 \times 10^{-7}$ & strong \\
\hline & & $B-I$ & $B$ & 52 & 0.42 & $2.1 \times 10^{-3}$ & strong \\
\hline \multirow[t]{3}{*}{2458420} & 20181028 & $R-V$ & $V$ & 68 & 0.70 & $2.8 \times 10^{-11}$ & strong \\
\hline & & $R-B$ & $B$ & 67 & 0.82 & $9.5 \times 10^{-18}$ & strong \\
\hline & & $V-B$ & $B$ & 67 & 0.64 & $4.6 \times 10^{-9}$ & strong \\
\hline 2458424 & 20181101 & $I-R$ & $R$ & 132 & 0.36 & $1.8 \times 10^{-6}$ & strong \\
\hline \multirow[t]{3}{*}{2458425} & 20181102 & $R-V$ & $V$ & 84 & 0.26 & $1.9 \times 10^{-2}$ & mild \\
\hline & & $R-B$ & $B$ & 84 & 0.58 & $7.7 \times 10^{-9}$ & strong \\
\hline & & $V-B$ & $B$ & 84 & 0.49 & $1.8 \times 10^{-6}$ & strong \\
\hline \multirow[t]{3}{*}{2458456} & 20181203 & $R-V$ & $V$ & 51 & 0.49 & $1.8 \times 10^{-1}$ & no \\
\hline & & $R-B$ & $B$ & 51 & 0.20 & $2.6 \times 10^{-1}$ & no \\
\hline & & $V-B$ & $B$ & 51 & 0.21 & $3.0 \times 10^{-4}$ & weak \\
\hline
\end{tabular}

similar color so the color changes of AGN are not affected by the host galaxy. Wierzcholska et al. (2015) did a long-term observation of the BL Lacertae object and argued that the BWB trend is less likely caused by the host galaxy if the color-magnitude diagram shows separate branches. It is believed that the BWB trend is originated from the emission regions of the jet. As the object gets brighter, more relativistic electrons will be accelerated and injected into the emission zone. The high energy photons from the synchrotron mechanism typically emerge sooner and closer to the shock front than the lower energy ones, thus causing color variations and larger variation amplitude at higher frequencies (Chiang \& Böttcher 2002; Fiorucci et al. 2004). According to Feng et al. (2020), the significance of BWB trend might be affected by the strength of variation, the BWB trend caused by the shock will be more significant during a weaker phase of variation and vice versa.

No time-lag has been detected in our observation. Wu et al. (2012) mentioned four key parameters that 
resolution and measurement accuracy. According to Table 1, our temporal resolution (less than 5 minutes) is much smaller than previous detected time lag. Three or four filters were used to observe BL Lacertae on 10 nights which provided us large wavelength separation. Small variation amplitude (JD2457697) and low measurement accuracy (JD2458420, JD2458424) might be the reason why we didn't detect time-lag. In addition, correlation analysis will fail to detect the time lag (if there is any) between featureless or monotonically brightening or darkening light curves, such as those on JD 2457696, 2458012, and 2458014.

In conclusion, BL Lacertae showed IDV in 23 light curves on 12 nights among our 14 nights observation during 2016-2018. We found the IDV amplitude of BL Lacertae is greater at higher frequencies. In addition, BL Lacertae shows BWB trend on most of the nights. Finally, no time-lag has been detected in our observation.

\section{ACKNOWLEDGEMENTS}

This work has been supported by the National Natural Science Foundation of China grants 11973017.

\section{References}

Abdo, A. A., Ackermann, M., Agudo, I., et al. 2010, ApJ, 716, 30

Agarwal, A., \& Gupta, A. C. 2015, MNRAS, 450, 541

Alexander, T. 1997, Is AGN Variability Correlated with Other AGN Properties? ZDCF Analysis of Small

Samples of Sparse Light Curves, ed. D. Maoz, A. Sternberg, \& E. M. Leibowitz, Vol. 218, Astronomical

Time Series, ed. D. Maoz, A. Sternberg, \& E. M. Leibowitz, Vol. 218, 163

Alexander, T. 2013, arXiv e-prints, arXiv:1302.1508

Bach, U., Villata, M., Raiteri, C. M., et al. 2006, A\&A, 456, 105

Bhatta, G., \& Webb, J. 2018, Galaxies, 6, 2

Böttcher, M., Marscher, A. P., Ravasio, M., et al. 2003, ApJ, 596, 847

Butuzova, M. S. 2021, Astroparticle Physics, 129, 102577

Carini, M. T., Miller, H. R., Noble, J. C., \& Goodrich, B. D. 1992, AJ, 104, 15

Chiang, J., \& Böttcher, M. 2002, ApJ, 564, 92

Ciprini, S., Tosti, G., Teräsranta, H., \& Aller, H. D. 2004, MNRAS, 348, 1379

de Diego, J. A., Polednikova, J., Bongiovanni, A., et al. 2015, AJ, 150, 44

Edelson, R. A., \& Krolik, J. H. 1988, ApJ, 333, 646

Epstein, E. E., Fogarty, W. G., Hackney, K. R., et al. 1972, ApJ, 178, L51

Fan, J. H., \& Lin, R. G. 1999, ApJS, 121, 131

Fan, J. H., Qian, B. C., \& Tao, J. 2001, A\&A, 369, 758

Feng, H.-C., Liu, H. T., Bai, J. M., et al. 2020, ApJ, 888, 30

Fiorucci, M., Ciprini, S., \& Tosti, G. 2004, A\&A, 419, 25

Fossati, G., Maraschi, L., Celotti, A., Comastri, A., \& Ghisellini, G. 1998, MNRAS, 299, 433

Gaskell, C. M., \& Peterson, B. M. 1987, ApJS, 65, 1

Gaur, H., Gupta, A., Bachev, R., et al. 2017, Galaxies, 5, 94 
Goyal, A., Mhaskey, M., Gopal-Krishna, et al. 2013, Journal of Astrophysics and Astronomy, 34, 273

Gu, M. F., Lee, C. U., Pak, S., Yim, H. S., \& Fletcher, A. B. 2006, A\&A, 450, 39

Gupta, A. C., \& Joshi, U. C. 2005, A\&A, 440, 855

Gupta, A. C., Gaur, H., Wiita, P. J., et al. 2019, AJ, 157, 95

Heidt, J., \& Wagner, S. J. 1996, A\&A, 305, 42

Hu, S. M., Wu, J. H., Zhao, G., \& Zhou, X. 2006, MNRAS, 373, 209

Ikejiri, Y., Uemura, M., Sasada, M., et al. 2011, PASJ, 63, 639

Kurtanidze, O. M., Richter, G. M., \& Nikolashvili, M. G. 2001, in Galaxies and their Constituents at the

Highest Angular Resolutions, ed. R. T. Schilizzi, Vol. 205, 82

Li, X.-P., Luo, Y.-H., Yang, H.-T., et al. 2018, Research in Astronomy and Astrophysics, 18, 150

Marscher, A. P. 2014, ApJ, 780, 87

Marscher, A. P., \& Gear, W. K. 1985, ApJ, 298, 114

Meng, N., Wu, J., Webb, J. R., Zhang, X., \& Dai, Y. 2017, MNRAS, 469, 3588

Meng, N., Zhang, X., Wu, J., Ma, J., \& Zhou, X. 2018, ApJS, 237, 30

Miller, J. S., \& Hawley, S. A. 1977, ApJ, 212, L47

Nesci, R., Maesano, M., Massaro, E., et al. 1998, A\&A, 332, L1

Nikolashvili, M. G., \& Kurtanidze, O. M. 2004, Nuclear Physics B Proceedings Supplements, 132, 205

Padovani, P., \& Giommi, P. 1995, MNRAS, 277, 1477

Papadakis, I. E., Boumis, P., Samaritakis, V., \& Papamastorakis, J. 2003, A\&A, 397, 565

Papadakis, I. E., Villata, M., \& Raiteri, C. M. 2007, A\&A, 470, 857

Peterson, B. M., Wanders, I., Horne, K., et al. 1998, PASP, 110, 660

Peterson, B. M., Ferrarese, L., Gilbert, K. M., et al. 2004, ApJ, 613, 682

Racine, R. 1970, ApJ, 159, L99

Raiteri, C. M., Villata, M., Capetti, A., et al. 2009, A\&A, 507, 769

Sadun, A. C., Asadi-Zeydabadi, M., Hindman, L., \& Moody, J. W. 2020, Galaxies, 8, 11

Sandrinelli, A., Covino, S., Treves, A., et al. 2018, A\&A, 615, A118

Scarpa, R., Urry, C. M., Padovani, P., Calzetti, D., \& O'Dowd, M. 2000, ApJ, 544, 258

Smith, P. S., Balonek, T. J., Heckert, P. A., Elston, R., \& Schmidt, G. D. 1985, AJ, 90, 1184

Speziali, R., \& Natali, G. 1998, A\&A, 339, 382

Stalin, C. S., Gopal-Krishna, Sagar, R., et al. 2006, MNRAS, 366, 1337

Vagnetti, F., \& Trevese, D. 2003, Mem. Soc. Astron. Italiana, 74, 963

Villata, M., Raiteri, C. M., Kurtanidze, O. M., et al. 2002, A\&A, 390, 407

Villata, M., Raiteri, C. M., Kurtanidze, O. M., et al. 2004a, A\&A, 421, 103

Villata, M., Raiteri, C. M., Aller, H. D., et al. 2004b, A\&A, 424, 497

Wagner, S. J., \& Witzel, A. 1995, ARA\&A, 33, 163

Webb, J. R., Freedman, I., Howard, E., et al. 1998, AJ, 115, 2244

Wierzcholska, A., Ostrowski, M., Stawarz, Ł., Wagner, S., \& Hauser, M. 2015, A\&A, 573, A69

Wu, J., Böttcher, M., Zhou, X., et al. 2012, AJ, 143, 108

Zhai, M., \& Wei, J. Y. 2012, A\&A, 538, A125 
Zhang, X., Wu, J., \& Meng, N. 2018, MNRAS, 478, 3513 